\title{
Clinical meaning of the World Health Organization morphologic classification (flat vs. tumoral) of gallbladder intraepithelial neoplasm as a prognostic factor in gallbladder cancer
}

\author{
Hongbeom Kim ${ }^{1 \#}$, Jin-Young Jang ${ }^{1 \#}$, Jihoon Chang ${ }^{1}$, Haeryoung Kim ${ }^{2}$, Yoonhyeong Byun ${ }^{1}$, Jae Ri Kim ${ }^{1}$, \\ Wooil Kwon ${ }^{1}$, Sun-Whe Kim ${ }^{1}$, Kyoung-Bun Lee ${ }^{2}$ \\ ${ }^{1}$ Department of Surgery and Cancer Research Institute, Seoul National University College of Medicine, Seoul, Korea; ${ }^{2}$ Department of Pathology, \\ Seoul National University College of Medicine, Seoul, Korea \\ Contributions: (I) Conception and design: SW Kim, H Kim, KB Lee; (II) Administrative support: W Kwon, JY Jang; (III) Provision of study materials \\ or patients: SW Kim, JY Jang, KB Lee, W Kwon, H Kim; (IV) Collection and assembly of data: H Kim, J Chang, Y Byun, JR Kim; (V) Data analysis \\ and interpretation: J Chang, JR Kim, Y Byun; (VI) Manuscript writing: All authors; (VII) Final approval of manuscript: All authors. \\ \#These authors contributed equally to this work. \\ Correspondence to: Kyoung-Bun Lee, MD, PhD. Department of Pathology, Seoul National University College of Medicine, Seoul National University \\ Hospital, 101 Daehak-ro, Jongno-gu, Seoul 110-744, Korea. Email: kblee@snuh.org.
}

Background: In the World Health Organization (WHO) classification, gallbladder (GB) intraepithelial lesions are grouped as flat or tumoral, according to their morphological features. The purpose of this study was to investigate the relationship between the morphologies and clinical features of GB cancer (GBC) and to examine the feasibility of using morphologic classification as a prognostic factor.

Methods: From January 2000 to December 2012, the available pathologic slide reviews of 381 patients were analyzed at the Seoul National University Hospital. All pathologic slides were evaluated by two pancreato-biliary tract pathology experts. GBCs were categorized into eight groups (Flat: F1-2, Borderline, Tumoral: Tu1-5), according to the thickness of the mucosal lesion, histologic patterns of the mucosa under microscopy, invasion extent, and patient history of premalignant lesions. According to the morphologic classification, clinical features were compared and survival analysis was performed.

Results: In three groups, flat lesions comprised 179 (46.9\%) cases and borderline and tumoral comprised 97 (25.4\%) and 105 (27.5\%) cases, respectively. More favorable pathologic and clinical results were found within the tumoral group. The borderline group had an intermediate tendency between flat and intraluminal in clinicopathologic parameters. In the curative resected T2 stage group, the borderline group demonstrated an intermediate trend compared to that of the flat and tumoral groups, but this was statistically insignificant $(\mathrm{P}=0.08)$.

Conclusions: Flat type GBCs show worse prognosis than tumoral GBCs. The morphological classifications between flat and tumoral on the basis of $1 \mathrm{~cm}$ and by papillary feature is feasible. Tumor morphology can be used as a reference while deciding the treatment plan, especially in T2 GBC.

Keywords: Gallbladder neoplasm; morphology; histology; classification; prognosis

Submitted Jan 09, 2020. Accepted for publication Aug 28, 2020.

doi: $10.21037 /$ atm-20-432

View this article at: http://dx.doi.org/10.21037/atm-20-432 


\section{Introduction}

To understand the characteristics of malignancy, it is important to understand the carcinogenic signaling pathway. There are two major carcinogenic pathways in gallbladder cancer (GBC) $(1,2)$. First is the dysplasiacarcinoma pathway, which resembles uterine cervix cancer. The other is adenoma-carcinoma sequence, which is similar to that of colorectal cancer. The majority of GBCs manifest via the dysplasia pathway, and the adenoma pathway accounts for approximately $5-10 \%$ in GBC $(1,3)$. In these pathways, cancer arises from a premalignant intraepithelial lesion. However, the definition and classification of an intraepithelial lesion in the gallbladder (GB) is ambiguous, and studies on its clinical features not been actively performed.

The World Health Organization (WHO) definition and classification of premalignancy is widely referenced. The WHO classifies the intraepithelial neoplasm of GB into adenoma (tubular, papillary, and tubulopapillay), biliary intraepithelial neoplasia, grade 3 (BilIN-3), intracystic papillary neoplasm (ICPN), and mucinous cystic neoplasm (4). Each subtype is categorized according to morphology and has its own clinical and pathologic features. The morphology of adenoma is typically polypoid, single, and well demarcated. A small portion of adenomas progress to invasive carcinomas $(5,6)$. ICPN is defined as an intracystic papillary neoplasm of the GB and is associated with invasive papillary carcinoma, which has different clinical characteristics than typical GBC (7). Each subtype is distinct, manifesting disease differently; however, they could be grouped instead into flat type or tumoral types, according to their gross and microscopic morphologies, similar to other biliary-pancreas tract neoplasms (Table 1) (8).

Adenoma and ICPN are included in the tumoral type. Even though the morphologies of the two subtypes are similar, there are no definitely reliable criteria to differentiate between adenoma and ICPN. It is especially difficult to distinguish between the papillary features of adenoma and ICPN. Therefore, a new definition of intracholecystic papillary tubular neoplasm (ICPTN) has been suggested. ICPTN is exophytic (papillary or polypoid) well-demarcated, with intramucosal GB masses measuring $\geq 1.0 \mathrm{~cm}$ (9). ICPTN could include adenoma and ICPN and exclude exuberant papillary hyperplasia with dysplasia according to the thickness of the mucosa $(<1 \mathrm{~cm})$. A determination of ICPTN offers a better prognosis than conventional GBC originating from BilIN-3. A new morphological definition attributed to the thickness of mucosa $\geq 1.0 \mathrm{~cm}$ shows that morphological classification has different clinical features (9).

Another morphological feature that provides a different prognosis is papillary appearance in the lesion. Papillary GB neoplasms show a favorable prognosis. However, the definition of papillary appearance is ambiguous. There is a discrepancy in the categorization of gross and microscopic papillary features. This confusion is more common in GB than other organs due to the histologic features of the normal GB mucosa, where papillary appearance is observed microscopically. Metaplasia and hyperplasia by recurrent inflammation are also observed in the normal GB mucosa (10). Thus, it is difficult to distinguish between a papillary neoplasm or a papillary change from the normal mucosa; and accordingly, papillary appearance needs to be clarified. Therefore, we divided papillary neoplasms according to the thickness of the mucosa into greater than $1 \mathrm{~cm}$ or less than $1 \mathrm{~cm}$.

As mentioned above, morphologic features (tumoral or flat, papillary) of the GB lesion are related to their clinical feature. To study the relationship between morphology and the corresponding clinical feature of GB cancer (GBC), the GBC lesions were grouped into flat or tumoral types, according to the thickness of mucosal portion of tumor $(1 \mathrm{~cm})$, macroscopic and microscopic morphologies (papillary or not), background premalignant lesions, and histology type of the associated invasive tumor. In the flat type, the borderline group was defined separately. In GBC, the borderline group has papillary features, but its mucosal height is not over $1 \mathrm{~cm}$. Therefore, the purpose of this study was to investigate the relationship between different morphologies (flat $v s$. tumoral) and clinical features of GBC and to examine the feasibility of using morphologic classification as a prognostic factor in GBC. We present the following study in accordance with the STROBE reporting checklist (available at http://dx.doi.org/10.21037/atm-20432).

\section{Methods}

\section{Patients and data collection}

From January 2000 to December 2012, 416 consecutive patients underwent surgery for GBC at the Seoul National University Hospital. Among them, 381 patients with pathologic slide reviews available were analyzed. Patient 
Table 1 Classification of intraepithelial neoplasms in the biliary-pancreatic tract

\begin{tabular}{lll}
\hline Organ & Flat & Tumoral \\
\hline Bile duct & BillN & Intraductal papillary neoplasm of bile duct (IPNB) \\
Gallbladder & BillN & Adenoma; intracystic papillary neoplasm (ICPN) \\
Ampulla of Vater & Flat intraepithelial neoplasia high grade & Intestinal type adenoma; non-invasive pancreatobiliary neoplasm \\
Pancreas & Pancreatic intraepithelial neoplasia (PanIN) & Intraductal papillary mucinous neoplasm (IPMN) \\
\hline
\end{tabular}

characteristics were reviewed for age, sex, pathologic diagnosis, operation method, adjuvant treatment, and recurrence. The clinical data for these patients were prospectively collected via electronic medical record data. Pathologic data were also reviewed for differentiation, presence of tubular type, invasive component, cell type, lymphatic invasion, vascular invasion, and perineural invasion.

\section{Pathologic classification}

All pathologic data, including new classifications, were evaluated by two pancreato-biliary tract specialized pathologists (Kyoung-Bun Lee and Haeryoung Kim). Two pathologists reviewed each of the same slides. When comparing each result with another result, the final classification was decided by discussing the slide again. GBCs were classified according to the morphology of the background intraepithelial lesion and were categorized into eight groups (Flat: F1-3, Tumoral: Tu1-5), and according to the extent of invasion, gross type, and mucosal changes. F1 was defined as minimally invasive carcinoma with flat intraepithelial lesions, which was invisible under gross inspection; had no mucosal hyperplasia and thickening; where the invasive tumor was observed only under microscopic examination ( $>40 \times$ power); or only intraepithelial carcinoma (carcinoma in situ) was observed. F2 was defined as a widely invasive carcinoma with and without flat intraepithelial lesions, the presence of nodular sclerotic gross lesions, without mucosal hyperplasia or papillary type dysplasia in the adjacent mucosa both upon macroscopic and microscopic inspection. F3 was defined as possessing papillary features with no dominating presence of polyps; and the mucosa also bore papillary features around the invasive tumor under microscopy, but the mucosal lesion did not have any polypoid lesions greater than $1 \mathrm{~cm}$ upon gross inspection. Microscopic features of the mucosa of F3 was micropapillary or short papillary epithelial dysplasia directly on the stroma, just above proper muscle or lamina propria, lacking a thick fibrovascular stalk. The F3 category is comprised of minimally invasive and widely invasive tumors. The borderline group was classified under F3 as it shows papillary features under the microscope, but it is ambiguous macroscopically. Tu1 was defined as demonstrating papillary features with polyps, which results in a polypoid mucosal lesion greater in 1 $\mathrm{cm}$ in height. $\mathrm{F} 3$ and Tu1 can be distinguished by height: whether the height is below $1 \mathrm{~cm}$ or above $1 \mathrm{~cm}$. Tu2 represents noninvasive papillary carcinoma, which has been described as noninvasive papillary carcinoma or biliary papillomatosis. Gross mucosa is replaced entirely by sessile papillary mucosa and is histologically composed of long slender papillary neoplasms with a fibro-vascular core. The major distinguishing factors between Tu1 and Tu2 was merely the observed spreading feature versus a localized polypoid feature. Invasive papillary carcinoma was classified as Tu3, which presents the features of Tu2 but expresses an invasive tumor. Tu4 and Tu5 were defined as minimally invasive adenocarcinomas arising in the adenoma as well as widely invasive carcinomas arising in the adenoma, respectively. The criteria differentiating between minimal and wide invasiveness was the same as for F1 and F2. The morphologic definition of adenoma adheres to the rules of the 2010 WHO classification, in which adenoma is defined as the proliferative epithelial lesion with tubular, tubulopapillary or papillary pattern and grossly identified as an isolated polypoid lesion. Among these patterns, the predominant papillary pattern was classified as Tu1, as mentioned before. These eight subgroups were classified from three groups: flat $(\mathrm{F} 1,2)$, borderline $(\mathrm{F} 3)$ and tumoral (Tu1-5); the key classifying criteria and representative pictures of gross and microscopic features under low and high magnifications are summarized in Table 2 and Figure 1.

\section{Statistical analysis}

All statistical analysis was performed using SPSS version 22.0 (IBM, Almank, NY, USA). Nominal variables were 
Table 2 Classification according to gross morphologic and histologic patterns

\begin{tabular}{lccccccc}
\hline Classification & Height & Papillary & Invasiveness & Spread direction & Premalignant lesion & Carcinogenic pathway & $\mathrm{n}$ (\%) \\
\hline F1 & $<1 \mathrm{~cm}$ & - & $\mathrm{M}$ & - & Bil IN & Metaplasia/dysplasia & 20 (5.2) \\
F2 & $<1 \mathrm{~cm}$ & - & W & - & Bil IN & Metaplasia/dysplasia & $159(41.7)$ \\
Borderline (F3) & $<1 \mathrm{~cm}$ & Papillary & M or W & - & ICPN & Unknown \\
Tu1 & $\geq 1 \mathrm{~cm}$ & Papillary & M & Upside & ICPN & Hyperplasia/dysplasia & 27 (7.1) \\
Tu2 & $\geq 1 \mathrm{~cm}$ & Papillary & M & Laterally & ICPN & Hyperplasia/dysplasia & 18 (4.7) \\
Tu3 & $\geq 1 \mathrm{~cm}$ & Papillary & W & - & ICPN & Hyperplasia/dysplasia & 18 (4.7) \\
Tu4 & $\geq 1 \mathrm{~cm}$ & - & M & - & Adenoma & Adenoma-carcinoma & 28 (7.3) \\
Tu5 & $\geq 1 \mathrm{~cm}$ & - & W & - & Adenoma & Adenoma-carcinoma & $14(3.7)$ \\
\hline
\end{tabular}

M, minimally; W, widely; Bil IN, biliary intraepithelial neoplasm; ICPN, intracholecystic papillary neoplasm.

compared using the Chi-squared test or Fisher's exact test, and continuous variables were compared using the Student's $t$-test or analysis of variance (ANOVA). Survival was determined using the Kaplan-Meier method with survival curves compared using the log-rank test. $\mathrm{P}$ values of less than 0.05 were considered statistically significant. As a prognostic factor, recurrence was defined through follow up image data such as computerized tomography, magnetic resonance imaging or elevated tumor markers, resulting in the patient undergoing additional chemotherapy. To determine recurrence risk factors the Cox regression test was used.

The study was conducted in accordance with the Declaration of Helsinki (as revised in 2013). The present study protocol was reviewed and approved by the Institutional Review Board of the Seoul National University Hospital (approval No. 1508-081-695). The process of obtaining informed consent was waived according to the decision of the IRB.

\section{Results}

\section{Demographics}

The mean age of the 381 patients was $67.4 \pm 10.8$ years. Two hundred and nine patients were female (54.8\%). Classifications of T1a, T1b, T2, T3, and T4 carcinoma were 44 (11.5\%), 52 (13.6\%), 23 (6.0\%), 161 (42.2\%), 86 $(22.5 \%)$, and $15(3.9 \%)$, respectively, as identified via in situ. Curative resections were performed on 298 patient samples $(78.2 \%)$. Cholecystectomies were performed in 191 patients $(50.1 \%)$ and extended cholecystectomies were performed in 165 patients (43.3\%). Hepatectomies; right hemihepatectomies or extended right hemihepatectomies were performed in 12 patients (3.1\%). There were eight pancreaticoduodenectomy cases $(2.1 \%)$ and five case of hepatopancreatic duodenectomies. Gallstones were present in 85 cases $(24.5 \%)$. The clinicopathological features of 381 patients were shown in Table 3.

The most common type of GBCs observed was F2, a widely invasive carcinoma with flat atypia $(\mathrm{n}=159,41.7 \%)$. The flat tumor types classified under F1 and F2 were combined as one ( $\mathrm{n}=169,49.9 \%)$; while the tumor types classified into Tu1, Tu2, Tu3, Tu4 and Tu5 ( $\mathrm{n}=105,27.6 \%)$ were classified as another. Finally, the F3 flat tumor type $(\mathrm{n}=97,25.5 \%)$ was separately considered; these were carcinomas with papillary dysplasia, lacking any visible dominant polyps, with either tumors that were either minimally invasive or invasive (Table 2).

\section{Clinicopathological features according to morphologic classification}

Table 3 shows clinicopathological features according to morphologic classification. There were no observable differences seen with age or sex between the classifications. The maximal diameter of a lesion was seen in the flat group compared to either the borderline or tumoral groups $(\mathrm{P}=0.06)$. In the flat group, more patients had advanced $\mathrm{T}$ stage disease. In addition, lymph node metastasis and distant metastasis were more common in the flat group than in either the borderline or tumoral groups. Curative resections were more frequently performed in the tumoral group than in the borderline or flat groups. Lastly, most of the patients in the borderline and tumoral groups underwent simple cholecystectomies or extended cholecystectomies. 

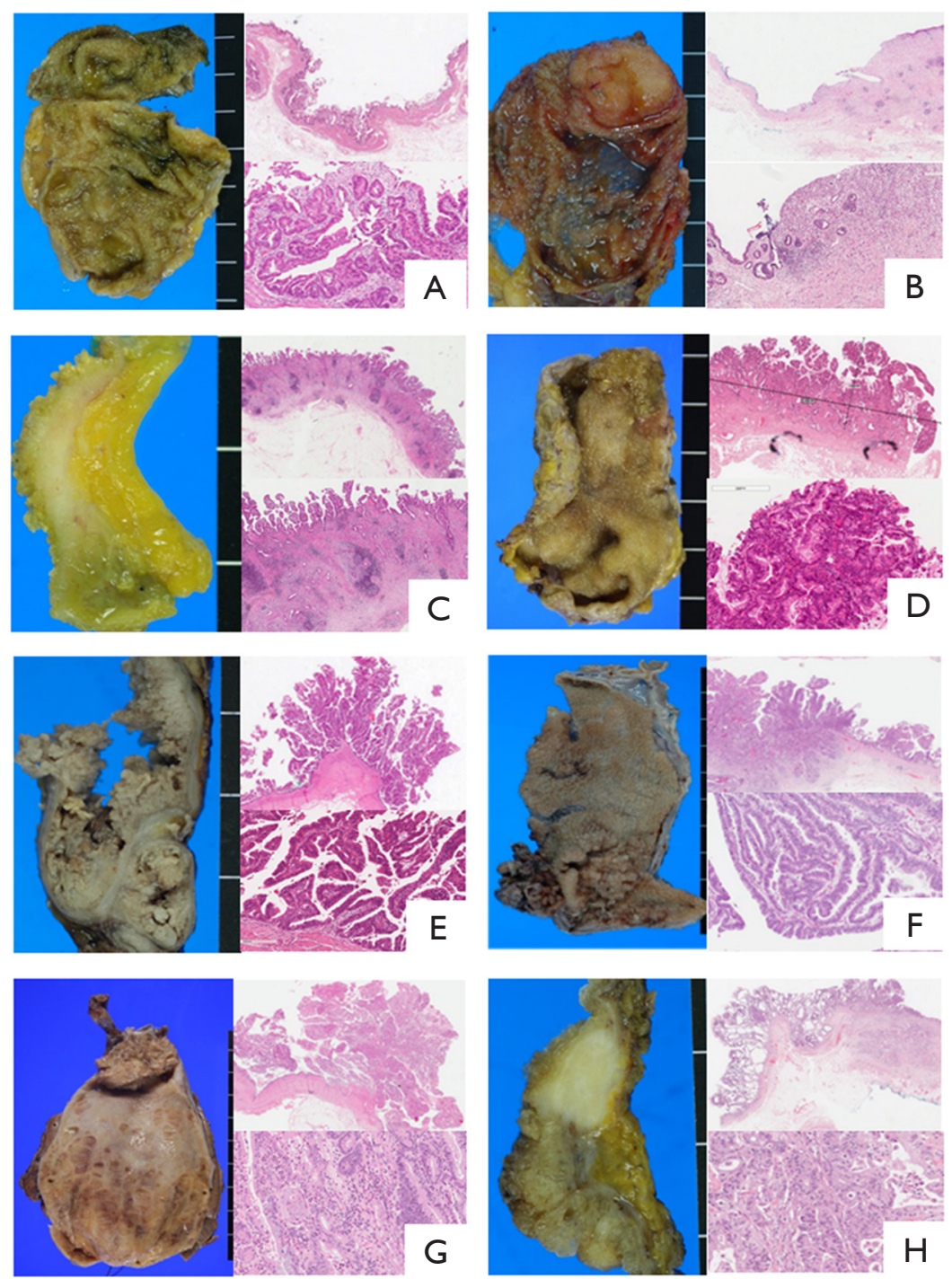

Figure 1 Classification according to gross morphologic and histologic. (A) F1: minimally invasive carcinoma with flat atypia; (B) F2: widely invasive carcinoma with flat atypia; (C) F3: carcinoma with papillary dysplasia but without any dominant polyps; (D) Tu1: carcinoma with papillary dysplasia and dominant polyp; (E) Tu2: noninvasive papillary carcinoma; (F) Tu3: invasive papillary carcinoma; (G) Tu4: minimally invasive adenocarcinoma arising in adenoma; (H) Tu5: widely invasive carcinoma arising in adenoma (left, gross; right upper, hematoxylin \& eosin $(\mathrm{HE}) \times 12.5$; right lower, $\mathrm{HE} \times 200)$.

The proportion of well differentiated cancer observed was $27.9 \%, 46.4 \%$ and $66.7 \%$ in the flat, borderline and tumoral groups, respectively $(\mathrm{P}<0.01)$. More invasive features such as lymphatic invasion, vascular invasion, perineural invasion were found in the flat group. Intestinal cell type of intraepithelial portion was higher in the borderline or tumoral lesions than in the flat lesion $(29.9 \%$, 25.75 , and $16.8 \%, \mathrm{P}=0.03)$. Histologic characteristics of dedifferentiation such as signet ring cell features, spindle cells, or solid pattern, were more frequently found in flat lesions than borderline or tumoral lesions (46.4\%, $17.5 \%$ and $11.4 \%, \mathrm{P}<0.01)$. The borderline group manifested intermediate histopathologic parameters of the flat and tumoral groups (Table 4).

\section{Survival and recurrence analysis}

The 5 -year survival rates were $58.5 \%$ in a total of 
Table 3 Demographic and clinicopathological characteristics of 381 patients

\begin{tabular}{|c|c|c|c|c|c|}
\hline Parameters & $N=381$ & Flat $(n=179, \%)$ & Borderline $(n=97, \%)$ & Tumoral $(n=105, \%)$ & $P$ value \\
\hline Sex & & & & & 0.149 \\
\hline Male & 172 & $90(50.3)$ & $41(42.3)$ & $41(39.0)$ & \\
\hline Female & 209 & $89(49.7)$ & $56(57.7)$ & $64(61.0)$ & \\
\hline pT & & & & & $<0.001$ \\
\hline Tis & 44 & $12(6.7)$ & $11(11.3)$ & $21(20.0)$ & \\
\hline T1a & 52 & $5(2.8)$ & $17(17.5)$ & $30(28.6)$ & \\
\hline $\mathrm{T} 1 \mathrm{~b}$ & 23 & $6(3.4)$ & $5(5.1)$ & $12(11.4)$ & \\
\hline $\mathrm{T} 4$ & 15 & $15(8.4)$ & $0(0.0)$ & $0(0.0)$ & \\
\hline pM & & & & & 0.052 \\
\hline MO & 347 & $157(87.7)$ & 89 (90.9) & $101(96.2)$ & \\
\hline M1 & 34 & $22(12.2)$ & $8(9.0)$ & $4(3.8)$ & \\
\hline $\mathrm{pN}$ & & & & & 0.002 \\
\hline No/x & 276 & $119(60.5)$ & $67(69.1)$ & $90(85.7)$ & \\
\hline $\mathrm{N} 1$ & 105 & $60(33.5)$ & $30(30.9)$ & 15 (14.3) & \\
\hline Curative resection & & & & & $<0.001$ \\
\hline Extended cholecystectomy & 165 & $82(45.8)$ & $35(36.1)$ & $48(45.7)$ & \\
\hline Hepatectomy & 12 & $11(6.1)$ & $1(1.0)$ & $0(0.0)$ & \\
\hline Pancreaticoduodenectomy & 8 & $6(3.4)$ & $1(1.0)$ & $1(1.0)$ & \\
\hline HPD & 5 & $5(2.8)$ & $0(0.0)$ & $0(0.0)$ & \\
\hline Presence of gallstone & 82 & $39(22.7)$ & $16(16.5)$ & $27(25.7)$ & 0.362 \\
\hline
\end{tabular}

Hepatectomy, right hemihepatectomy or extended right hemihepatectomy; HPD, hepatopancreaticoduodenectomy.

381 patients and $72.0 \%$ in the curative resected group. Moreover, the 5-year disease-free survival rate were $66.2 \%$, $74.9 \%$ and $16.8 \%$ in the entire, curative and palliative groups, respectively. In the curative resected group, the recurrence rate differed according to the $\mathrm{T}$ stage and morphologic classification; both were statistically significant $(\mathrm{P}<0.01, \mathrm{P}<0.01)$.
Table 5 shows the risk factors of the curative resected GBC patients. Univariate analysis indicates that the following are risk factors: $\mathrm{T}$ stage, $\mathrm{N}$ stage, morphologic classification, differentiation, vascular invasion, lymphatic invasion, perineural invasion and adjuvant chemo therapy. In the multivariate analysis, the $\mathrm{T}$ stage was the only significant recurrence risk factor for curative resected GBC 
Table 4 Histopathologic features of GB cancers according to classification

\begin{tabular}{|c|c|c|c|c|c|}
\hline Parameters & $\mathrm{N}=381$ & Flat $(n=179, \%)$ & Borderline $(\mathrm{n}=97, \%)$ & Tumoral $(n=105, \%)$ & $P$ value \\
\hline Well & 165 & $50(27.9)$ & $45(46.4)$ & $70(66.7)$ & \\
\hline Moderate & 131 & $61(34.1)$ & $41(42.3)$ & $29(27.5)$ & \\
\hline Poor & 85 & $68(38.0)$ & $11(11.3)$ & $6(1.6)$ & \\
\hline Absent & 138 & $63(35.2)$ & $26(26.8)$ & $49(46.7)$ & \\
\hline Present & 243 & $116(64.8)$ & $71(73.2)$ & $56(53.3)$ & \\
\hline Dedifferentiated component $^{\dagger}$ & & & & & $<0.001$ \\
\hline Absent & 270 & $96(53.6)$ & $80(82.5)$ & $93(88.6)$ & \\
\hline Intestinal & 86 & $30(16.8)$ & $29(29.9)$ & $27(25.7)$ & \\
\hline Nonintentional & 295 & 149(83.2) & $68(70.1)$ & $78(74.3)$ & \\
\hline Lymphatic invasion & & & & & $<0.001$ \\
\hline Absent & 253 & $97(54.2)$ & $68(70.1)$ & $88(83.8)$ & \\
\hline Present & 128 & $82(45.8)$ & $29(29.9)$ & $17(16.1)$ & \\
\hline Vascular invasion & & & & & $<0.001$ \\
\hline Absent & 323 & 138(77.1) & $83(85.6)$ & $101(97.1)$ & \\
\hline Present & 58 & $41(22.9)$ & $14(14.4)$ & $3(2.9)$ & \\
\hline Absent & 258 & $151(84.4)$ & $53(54.6)$ & $54(51.4)$ & \\
\hline Present & 123 & 28 (15.6) & $44(45.4)$ & $51(48.6)$ & \\
\hline
\end{tabular}

\footnotetext{
${ }^{\dagger}$, signet ring cell, spindle cells, solid pattern.
}

patients $(\mathrm{P}<0.01)$.

To determine the effects of morphology, further analysis according to the $\mathrm{T}$ stage was performed. Within the $\mathrm{T} 1$ group, there was no recurrence within 5 years. There was no difference within the distinct morphologic classifications of the T3/4 advanced GBC group. However, in the $\mathrm{T} 2$ group the five-year disease-free survival rates were $79.2 \%, 67.4 \%$ and $59.6 \%$ in the tumoral, borderline and flat groups, respectively. The DFS rate tended to be lower according to the morphologic type, but there was no statistical significance $(\mathrm{P}=0.08)$ (Figure 2).

\section{Discussion}

There are many prognostic factors in GBC; one of them is macroscopic or microscopic morphology (11-13). This study was designed to deal with two questions. The first question was about papillary feature. The cancer which shows papillary morphologic feature offers excellent prognosis (14). However, the definition of papillary 

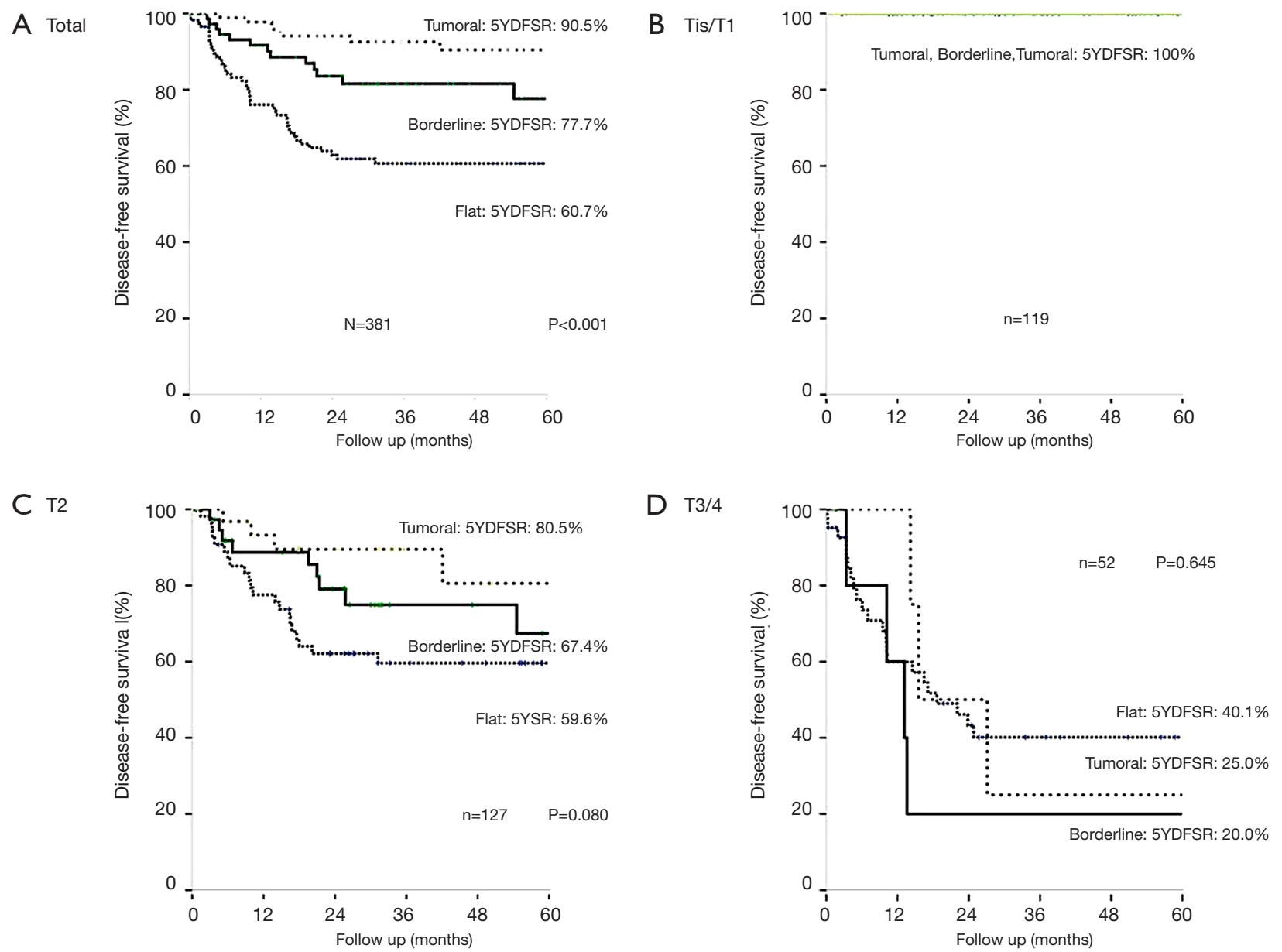

Figure 2 Disease-free survival (DFS) rates according to T classification subgroups. (A) DFS of total patients (n=381); (B) no event in Tis/T1 stage $(n=119)$; (C) DFS in pT2 stage ( $n=127)$; (D) DFS in pT3/4 stages $(n=52)$.

is ambiguous, 'What is the papillary? Microscopically or macroscopically?' Normal GB mucosa has papillary folding, and this mucosal folding is accentuated when there is recurrent inflammation (10). This increased papillary folding is referred to as papillary hyperplasia; and when the covered epithelial cells have signs of dysplasia such as stratification, increased nuclear atypia and pleomorphism, increased mitosis, etc., there is no standard for labeling this lesion as dysplasia in papillary hyperplasia of a papillary neoplasm. Accordingly, this makes it difficult to define a papillary lesion. The first aim of this study was to clarify papillary GBC. The second question was to determine whether there is any difference of prognosis between flat and tumoral types in GBC, and the feasibility of height (i.e., $1 \mathrm{~cm}$ ) as a determinant of polypoid lesion. To reach these objectives, we defined flat and tumoral types according to a height of $1 \mathrm{~cm}$ as the cutoff, referring to the WHO classification and Aday's definition of ICPTN $(4,9)$. Next, we looked to identify the disease subgroup (borderline), which manifested characteristics that fell in between tumoral (Tu1) and flat (F2) subgroups.

This study was a large-scale single-institute study. GBC was subdivided into 3 groups. In the flat type GBC, the lesions were commonly found in the advanced stage and curative resection rates were lower than in the tumoral type. Tumor morphology as well as the size of the lesion, when the GB mass is discovered, needs to be consideration factors for subsequent treatment. We define borderline as something too difficult to be included within ICPN, but which demonstrates papillary features with microscopic findings. The prognosis of the borderline zone is intermediate (between that of flat and tumoral). Of note, 
Table 5 Recurrence risk factors in GBC patients following curative resection

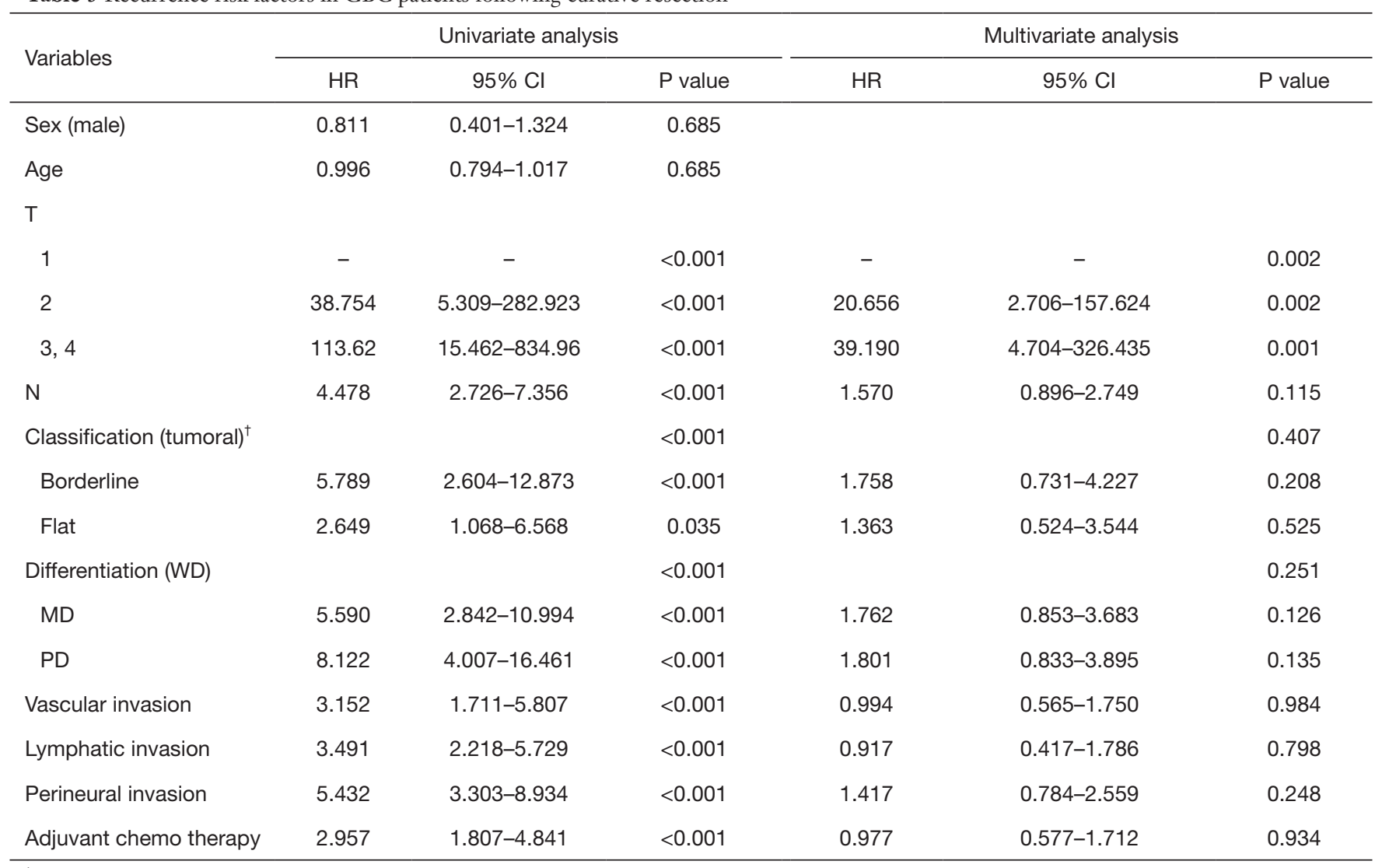

${ }^{\dagger}$, dominant polyp: $\geq 1 \mathrm{~cm}$ in height.

the borderline group is more similar to flat than to tumoral lesions. The T2 stage group demonstrated a recurrencefree survival rate similar to that of the borderline group, falling in the middle of the flat and tumoral types. Although the lesion bore papillary-like characteristics, its length fell below $1 \mathrm{~cm}$; and therefore, the group exhibited different characteristics from general papillary lesions.

In 2010, the WHO classified the premalignant lesion of GB into adenoma (tubular, papillary, tubulopapillay), biliary intraepithelial neoplasia, grade 3 (BilIN-3), intracystic papillary neoplasm, and mucinous cystic neoplasm (4). These lesions could also be grouped as flat to reflect the following: BilIN-3 or luminal lesions; adenoma, intracystic papillary neoplasm, and mucinous cystic neoplasm. However, there is no reliable criteria between adenoma and intracystic papillary neoplasm. Therefore, Adsay et al. (9) have suggested a new definition (ICPTN), which encompasses adenoma and intracystic papillary neoplasm that is characterized by an exophytic well-demarcated massforming tumor greater than $1 \mathrm{~cm}$. The makeup of ICPTN is papillary (43\%), tubulopapillary (31\%) and tubular (26\%). The predominant cell lineage patterns of morphology, supported by specific immunohistochemical markers, were biliary in $50 \%$, gastric foveolar in $16 \%$, gastric pyloric in $20 \%$, intestinal in $8 \%$, and oncocytic in $6 \%$. The factors associated with invasiveness were the extent of high-grade dysplasia, cell type, and papilla formation. Invasive ICPTN has a significantly better overall prognosis than others.

In our study, we tried to incorporate various criteria and terminologies of papillary lesions that have been argued by pathologists over time (Tu1-5). Accordingly, we compared these entities with flat lesions (F1-2) as well as an ambiguous entity that falls in the middle of flat and tumoral lesions (borderline, F3). Interestingly, the F3 group exhibited intermediate clinicopathologic features resembling something in between that of flat and tumoral lesions. In this group, the stage and tumor progression resembled more closely to those of a flat lesion, but histological features were more similar to those of a tumoral lesion, even though the invasive portion was similar to a flat lesion. 
A possible mechanism could perhaps be that dysplasia in the flat type lesion occurs via a hyperplastic process, although further studies are needed to confirm this hypothesis.

"Papillary" is a broadly used terminology in anatomic pathology. The general concept of papillary is that of a leaf-like structure composed of epithelial cells with or without the presence of a fibro-vascular core. Depending on the size of papillae, papillary lesions have opposite clinicopathological meanings. In the papillary lesion that is only visible by microscopy at high magnification, fibrovascular cores are scant or absent, and epithelial cells are directly budded on underlying the stromal tissue. This type is usually labeled as "micropapillary," and has been reported as a histologic indication of poor prognosis in several invasive carcinomas (15-18). In contrast, papillary lesions described at the macroscopic level or at a low microscopic magnification have a thick fibro-vascular core or form sessile or stalked polypoid lesions. This papillary lesion is similarly classified as papillary neoplasm in the pancreatobiliary organ and exhibits indolent biologic behavior and has better prognosis than a non-papillary neoplasm. The use of this mixed terminology may be one of the reasons for the few reports on the papillary neoplasm. Therefore, the morphologic criterion defining a papillary neoplasm need to be refined.

A similar spectrum of lesions exists in another biliarypancreas tract. Pancreatic intraepithelial neoplasia (PanIN) and intraductal papillary mucinous neoplasm (IPMN) are premalignant lesion in the pancreas. Recently, a similar finding was observed in the ampulla of Vater: intraepithelial neoplasms intra-ampullary papillary-tubular neoplasia (IAPNs) (8). These lesions have been grouped into flat and tumoral types (Table 1). The characteristics of flat lesions include grossly unidentifiable and non-mucinous tumor types. As the change occurs, shifting to malignancy, the lesion usually shows a tubular structure and leads to a poor prognosis. In contrast, tumoral types are generally identifiable and mucinous and have a better prognosis than do flat lesions.

Cell lineage also differs between the two groups: nonintestinal type (pancreatobiliary, gastric and oncocytic) in the flat, and intestinal type in the tumoral type. Subsequent immunohistochemical analysis yielded distinctive results based on tumor type. MUC1 is usually positive in pancreatobiliary differentiation (pancreatic IPMNs, ampullary IAPNs) (19-21). MUC2 and CDX2 are positive in intestinal differentiation $(8,21)$, MUC6 is pyloric marker and MUC5AC is foveolar mucin marker (22). Flat and tumoral types appear to different in the carcinogenesis pathway. The flat premalignant lesions follow a metaplasiacarcinoma sequence while the tumoral type follows an adenoma-carcinoma sequence $(23,24)$. Thus, the flat and tumoral types are unique and should be considered as separate entities.

The treatment strategy for GBCs, including the feasibility of laparoscopic surgery, extent of liver resection, adjuvant therapy, and regimen of chemo therapy, differs according to the T stage. For surgery, in T1 GBCs, a simple cholecystectomy may be undertaken, or even laparoscopic cholecystectomy is feasible, according to some reports (25). However, in T2 cancer, there is still some controversy as to the extent of surgery and adjuvant treatment plans that should be followed (26). Therefore, studies on T2 GBC have been actively undertaken (27-29). One of them examines tumor location ("hepatic side" or "peritoneal side"). Hepatic side T2 GBC offers a poor prognosis compared to the peritoneal side $(30,31)$. Thus, we believe tumor morphology could be another influencing factor like tumor location and tumor stage.

\section{Conclusions}

In conclusion, flat type GBCs are associated with a worse prognosis than tumoral GBCs. Thus, unusual thickening of the GB wall, which may lead to malignancy, needs to be assessed with care. Morphologic classifications of flat or tumoral on the basis of length $(1 \mathrm{~cm})$ and papillary features are feasible. Additionally, the borderline group possesses papillary features as well as a length of $1 \mathrm{~cm}$; yet, overall, the group reflects the characteristics of the disease manifested by the flat type GBC. The most powerful prognostic factor is the $\mathrm{T}$ stage in GBC. Thus, tumor morphology can be utilized as a reference when deciding on the treatment plan, especially in T2 GBC.

\section{Acknowledgments}

Funding: This study was supported by the Seoul National University Hospital (SNUH) Research Fund (Grant number 04-2015-3110).

\section{Footnote}

Reporting Checklist: The authors present the study in accordance with the STROBE reporting checklist. Available at http://dx.doi.org/10.21037/atm-20-432 
Data Sharing Statement: Available at http://dx.doi. org/10.21037/atm-20-432

Conflicts of Interest: All authors have completed the ICMJE uniform disclosure form (available at http://dx.doi. org/10.21037/atm-20-432). All authors have no conflicts of interest to declare.

Ethical Statement: The authors are accountable for all aspects of the work in ensuring that questions related to the accuracy or integrity of any part of the work are appropriately investigated and resolved. The study was conducted in accordance with the Declaration of Helsinki (as revised in 2013). The present study protocol was reviewed and approved by the Institutional Review Board of the Seoul National University Hospital (approval No. 1508081-695). The process of obtaining informed consent was waived according to the decision of the IRB.

Open Access Statement: This is an Open Access article distributed in accordance with the Creative Commons Attribution-NonCommercial-NoDerivs 4.0 International License (CC BY-NC-ND 4.0), which permits the noncommercial replication and distribution of the article with the strict proviso that no changes or edits are made and the original work is properly cited (including links to both the formal publication through the relevant DOI and the license). See: https://creativecommons.org/licenses/by-nc-nd/4.0/.

\section{References}

1. Wistuba II, Gazdar AF. Gallbladder cancer: lessons from a rare tumour. Nat Rev Cancer 2004;4:695-706.

2. Albores-Saavedra J, Chablé-Montero F, González-Romo MA, et al. Adenomas of the gallbladder. Morphologic features, expression of gastric and intestinal mucins, and incidence of high-grade dysplasia/carcinoma in situ and invasive carcinoma. Hum Pathol 2012;43:1506-13.

3. Jang KT, Ahn S. Tumoral versus flat intraepithelial neoplasia of pancreatobiliary tract, gallbladder, and ampulla of Vater. Arch Pathol Lab Med 2016;140:429-36.

4. Bosman FT, Carneiro F, Hruban RH, et al. WHO classification of tumours of the digestive system (4th eds). World Health Organization, 2010.

5. Albores-Saavedra J, Vardaman CJ, Vuitch F. Nonneoplastic polypoid lesions and adenomas of the gallbladder. Pathol Annu 1993;28:145-77.

6. Kijima $\mathrm{H}$, Watanabe $\mathrm{H}$, Iwafuchi $M$, et al. Histogenesis of gallbladder carcinoma from investigation of early carcinoma and microcarcinoma. Acta Pathol Jpn 1989;39:235-44.

7. Hoang MP, Murakata LA, Katabi N, et al. Invasive papillary carcinomas of the extrahepatic bile ducts: a clinicopathologic and immunohistochemical study of 13 cases. Mod Pathol 2002;15:1251-8.

8. Ohike N, Kim GE, Tajiri T, et al. Intra-ampullary papillary-tubular neoplasm (IAPN): characterization of tumoral intraepithelial neoplasia occurring within the ampulla: a clinicopathologic analysis of 82 cases. Am J Surg Pathol 2010;34:1731-48.

9. Adsay V, Jang KT, Roa JC, et al. Intracholecystic papillarytubular neoplasms (ICPN) of the gallbladder (neoplastic polyps, adenomas, and papillary neoplasms that are $\geq 1.0$ $\mathrm{cm})$ : clinicopathologic and immunohistochemical analysis of 123 cases. Am J Surg Pathol 2012;36:1279-301.

10. Ross MH, Pawlina W. Histology: a Text and Atlas, with Correlated Cell and Molecular Biology (6th eds). Lippincott Williams \& Wilkins, 2010.

11. Goldin RD, Roa JC. Gallbladder cancer: a morphological and molecular update. Histopathology 2009;55:218-29.

12. Roa I, de Aretxabala X, Araya JC, et al. Morphological prognostic elements in gallbladder cancer. Rev Med Chil 2002;130:387-95.

13. Cariati A, Piromalli E, Cetta F. Gallbladder cancers: associated conditions, histological types, prognosis, and prevention. Eur J Gastroenterol Hepatol 2014;26:562-9.

14. Henson DE, Albores-Saavedra J, Corle D. Carcinoma of the gallbladder. Histologic types, stage of disease, grade, and survival rates. Cancer 1992;70:1493-7.

15. Fujita T, Konishi M, Gotohda N, et al. Invasive micropapillary carcinoma of the ampulla of Vater with extensive lymph node metastasis: Report of a case. Surg Today 2010;40:1197-200.

16. Yoshida Y, Nitadori JI, Shinozaki-Ushiku A, et al. Micropapillary histological subtype in lung adenocarcinoma of $2 \mathrm{~cm}$ or less: impact on recurrence and clinical predictors. Gen Thorac Cardiovasc Surg 2017;65:273-79.

17. Kumari N, Jha A, Vasudeva P, et al. High-grade urothelial carcinoma of bladder transforming to micropapillary variant on follow-up. Iran J Med Sci 2017;42:318-21.

18. Kim HJ, Park K, Kim JY, et al. Prognostic significance of a micropapillary pattern in pure mucinous carcinoma of the breast: comparative analysis with micropapillary carcinoma. J Pathol Transl Med 2017;51:403-9.

19. Adsay NV, Merati K, Basturk O, et al. Pathologically and 
biologically distinct types of epithelium in intraductal papillary mucinous neoplasms: delineation of an "intestinal" pathway of carcinogenesis in the pancreas. Am J Surg Pathol 2004;28:839-48.

20. Yonezawa S, Taira M, Osako M, et al. MUC1 mucin expression in invasive areas of intraductal papillary mucinous tumors of the pancreas. Pathol Int 1998;48:319-22.

21. Yonezawa S, Goto M, Yamada N, et al. Expression profiles of MUC1, MUC2, and MUC4 mucins in human neoplasms and their relationship with biological behavior. Proteomics 2008;8:3329-41.

22. Basturk O, Khayyata S, Klimstra DS, et al. Preferential expression of MUC6 in oncocytic and pancreatobiliary types of intraductal papillary neoplasms highlights a pyloropancreatic pathway, distinct from the intestinal pathway, in pancreatic carcinogenesis. Am J Surg Pathol 2010;34:364-70.

23. Bedenne L, Faivre J, Boutron MC, et al. Adenomacarcinoma sequence or "de novo" carcinogenesis? A study of adenomatous remnants in a population-based series of large bowel cancers. Cancer 1992;69:883-8.

24. Duarte I, Llanos O, Domke H, et al. Metaplasia and precursor lesions of gallbladder carcinoma. Frequency, distribution, and probability of detection in routine histologic samples. Cancer 1993;72:1878-84.

Cite this article as: Kim H, Jang JY, Chang J, Kim H, Byun Y, Kim JR, Kwon W, Kim SW, Lee KB. Clinical meaning of the World Health Organization morphologic classification (flat vs. tumoral) of gallbladder intraepithelial neoplasm as a prognostic factor in gallbladder cancer. Ann Transl Med 2020;8(21):1413. doi: $10.21037 / \mathrm{atm}-20-432$
25. Jang JY, Heo JS, Han Y, et al. Impact of type of surgery on survival outcome in patients with early gallbladder cancer in the era of minimally invasive surgery:oncologic safety of laparoscopic surgery. Medicine 2016;95:e3675.

26. Horgan AM, Amir E, Walter T, et al. Adjuvant therapy in the treatment of biliary tract cancer: a systematic review and meta-analysis. J Clin Oncol 2012;30:1934-40.

27. Jung W, Jang JY, Kang MJ, et al. Effects of surgical methods and tumor location on survival and recurrence patterns after curative resection in patients with $\mathrm{T} 2$ gallbladder cancer. Gut Liver 2016;10:140-6.

28. Wakai T, Shirai Y, Yokoyama N, et al. Depth of subserosal invasion predicts long-term survival after resection in patients with T2 gallbladder carcinoma. Ann Surg Oncol 2003;10:447-54.

29. Chijiiwa K, Nakano K, Ueda J, et al. Surgical treatment of patients with $\mathrm{T} 2$ gallbladder carcinoma invading the subserosal layer. J Am Coll Surg 2001;192:600-7.

30. Lee H, Choi DW, Park JY, et al. Surgical Strategy for T2 Gallbladder Cancer According to Tumor Location. Ann Surg Oncol 2015;22:2779-86.

31. Shindoh J, de Aretxabala X, Aloia TA, et al. Tumor location is a strong predictor of tumor progression and survival in T2 gallbladder cancer: an international multicenter study. Ann Surg 2015;261:733-9. 